\title{
On some common fixed point theorems with PPF dependence in Banach spaces
}

\author{
Bapurao C. Dhage $e^{a, *}$ \\ ${ }^{a}$ Kasubai, Gurukul Colony, Ahmedpur-413 515, Dist. Latur, Maharashtra, India \\ This paper is dedicated to Professor Ljubomir Ćirić \\ Communicated by Professor V. Berinde
}

\begin{abstract}
In this paper, some results concerning the existence of common fixed points, coincidence points and approximating fixed points with PPF dependence for the pairs of operators in Banach spaces satisfying a generalized contractive condition are proved. The novelty of the present work lies in the fact that the domain and the range spaces of the operators in questions are not same and all the results are obtained via constructive methods. Our results generalize and extend the fixed point theorems with PPF dependence of Bernfeld et al. [S. R. Bernfeld, V. Lakshmikatham and Y. M. Reddy, Applicable Anal. 6 (1977), 271-280] and Dhage [B. C. Dhage, Fixed point Theory, (to appear)] under more general contractive conditions.(C)2012 NGA. All rights reserved.
\end{abstract}

Keywords: Banach space; Fixed point theorem; PPF dependence.

2010 MSC: Primary 34K10; Secondary 47H10.

\section{Introduction}

In a recent paper [1], the authors proved some fixed point theorems for nonlinear operators in Banach spaces, where the domain and range of the operators are not same. The fixed point theorems of this kind are called PPF dependent fixed point theorems or the fixed point theorems with PPF dependence. Some basic fixed point theorems along this line such as those established in Bernfeld et al. [1] and Dhage [4] are useful for proving the solutions of nonlinear functional differential and integral equations which may depend

\footnotetext{
${ }^{*}$ Corresponding author

Email address: bcdhage@yahoo.co.in, bcdhage@gmail.com (Bapurao C. Dhage)
} 
upon the past history, present data and future consideration. The properties of a special Razumikhin class of functions are employed in the development of fixed point theory with PPF dependence in abstract spaces.

The topic of common fixed point theorem for pairs or families of contractive mappings in metric and abstract spaces is of great interest and has already been studied in the literature since long time. It seems that theory of common fixed point theorems has reached its culmination point and there are a good number of common fixed point theorems available for commuting as well as noncommuting mappings in metric spaces satisfying different contractive conditions. However, to the best of knowledge there no any result proved so far in the literature concerning the common fixed point theorems for the mappings in abstract spaces with different domain and range spaces. In the present paper, some common fixed point theorems with PPF dependence are proved for pairs of operators in Banach spaces satisfying generalized contractive conditions. We claim that our results of this paper are new and generalize some known basic results those proved in Bernfeld et al. [1] under more general contractive conditions.

\section{Preliminaries}

Given a Banach space $E$ with norm $\|\cdot\|_{E}$ and given a closed interval $I=[a, b]$ in $\mathbb{R}$, we consider the Banach space $E_{0}=C(I, E)$ of continuous $E$-valued functions on $I$. We equip the space $E_{0}$ with the supremum norm $\|\cdot\|_{E_{0}}$ defined as

$$
\|\phi\|_{E_{0}}=\sup _{t \in I}\|\phi(t)\|_{E} .
$$

For a fixed point $c \in I$, the Razumikhin class of functions(cf. [1, 4]) in $E_{0}$ is defined as

$$
\mathcal{R}_{c}=\left\{\phi \in E_{0} \mid\|\phi\|_{E_{0}}=\|\phi(c)\|_{E}\right\} .
$$

Let $T: E_{0} \rightarrow E$. A point $\phi^{*} \in E_{0}$ is called a PPF fixed point of $T$ if $T \phi^{*}=\phi^{*}(c)$ for some $c \in I$.

It is known that Razumikhin class of functions plays a significant role in proving the existence of PPFfixed points with different domain and range of the operators. See Bernfeld et al. [1], Dhage [4] and the references therein. Below we give different classes of contractive mappings for having common fixed point theorems with PPF dependence in Banach spaces.

Definition 2.1. An operator $T: E_{0} \rightarrow E$ is called Banach type contraction if there is a real number $0<\alpha<1$ such that

$$
\|T \phi-T \xi\|_{E} \leq \alpha\|\phi-\xi\|_{E_{0}}
$$

for all $\phi, \xi \in E_{0}$.

The following fixed point theorem with PPF dependence are proved respectively in Bernfield et al. [1] and Dhage [4].

Theorem 2.2 (Bernfeld et al. [1]). Suppose that $T: E_{0} \rightarrow E$ is a Banach type contraction. Then the following statements hold.

(a) If $\mathcal{R}_{c}$ is algebraically closed w.r.t the difference, then for a given $\phi_{0} \in E_{0}$ and $c \in[a, b]$, every sequence $\left\{\phi_{n}\right\}$ of iterates of $T$ defined by

$$
\left.\begin{array}{c}
T \phi_{n}=\phi_{n+1}(c) \\
\left\|\phi_{n}-\phi_{n+1}\right\|_{E_{0}}=\left\|\phi_{n}(c)-\phi_{n+1}(c)\right\|_{E}
\end{array}\right\}
$$

for $n-0,2, \ldots$; converges to a PPF fixed point of $T$. 
(b) If $\phi_{0}, \xi_{0} \in E_{0}$ and $\left\{\phi_{n}\right\},\left\{\xi_{n}\right\}$ are sequences defined by (2.4). Then,

$$
\left\|\phi_{n}-\xi_{n}\right\|_{E_{0}} \leq \frac{1}{1-\alpha}\left[\left\|\phi_{0}-\phi_{1}\right\|_{E_{0}}+\left\|\xi_{0}-\xi_{1}\right\|_{E_{0}}\right]+\left\|\phi_{0}-\xi_{0}\right\|_{E_{0}} .
$$

If, in particular $\phi_{0}=\xi_{0}$ and $\left\{\phi_{n}\right\} \not \equiv\left\{\xi_{n}\right\}$, then

$$
\left\|\phi_{n}-\xi_{n}\right\|_{E_{0}} \leq \frac{2}{1-\alpha}\left\|\phi_{0}-\xi_{0}\right\|_{E_{0}}
$$

(c) If $\mathcal{R}_{c}$ is topologically closed, then $T$ has a unique fixed point in $\mathcal{R}_{c}$.

The following definition is introduced in the literature on the lines of classical definition for contraction mappings given by Kannan [6].

Definition 2.3. An operator $T: E_{0} \rightarrow E$ is called strong Kannan type contraction if

$$
\|T \phi-T \xi\|_{E} \leq \alpha\left[\|\phi(c)-T \phi\|_{E}+\|\xi(c)-T \xi\|_{E}\right]
$$

for all $\phi, \xi \in E_{0}$ and some $c \in[a, b]$, where $0<\alpha<1 / 2$.

The following PPF dependent fixed point theorem is proved in Dhage [4].

Theorem 2.4 (Dhage [4). Suppose that $T: E_{0} \rightarrow E$ is a strong Kannan type contraction. Then the following statements hold.

(a) If $\mathcal{R}_{c}$ is algebraically closed w.r.t the difference, then for a given $\phi_{0} \in E_{0}$ and $c \in[a, b]$, every sequence $\left\{\phi_{n}\right\}$ of iterates of $T$ defined by 2.4 converges to a PPF fixed point of $T$.

(b) If $\phi_{0}, \xi_{0} \in E_{0}$ and $\left\{\phi_{n}\right\},\left\{\xi_{n}\right\}$ are sequences defined by (2.4). Then,

$$
\left\|\phi_{n}-\xi_{n}\right\|_{E_{0}} \leq\left(\frac{1-\alpha}{1-2 \alpha}\right)\left[\left\|\phi_{0}-\phi_{1}\right\|_{E_{0}}+\left\|\xi_{0}-\xi_{1}\right\|_{E_{0}}\right]+\left\|\phi_{0}-\xi_{0}\right\|_{E_{0}} .
$$

If, in particular $\phi_{0}=\xi_{0}$ and $\left\{\phi_{n}\right\} \not \equiv\left\{\xi_{n}\right\}$, then

$$
\left\|\phi_{n}-\xi_{n}\right\|_{E_{0}} \leq\left[\frac{2(1-\alpha)}{1-2 \alpha}\right]\left\|\phi_{0}-\xi_{0}\right\|_{E_{0}} .
$$

(c) If $\mathcal{R}_{c}$ is topologically closed, then $T$ has a unique fixed point in $\mathcal{R}_{c}$.

Now we list some of our observations.

Observation I The statement (a) in above Theorem presupposes that the Razumikhin class $\mathcal{R}_{c}$ of functions in $E_{0}$ is algebraically closed with respect to difference, that is, $\phi-\xi \in \mathcal{R}_{c}$ whenever $\phi, \xi \in \mathcal{R}_{c}$. Otherwise the construction of the sequence $\left\{\phi_{n}\right\}$ made there is not possible, because of the fact that

$$
\|\phi-\xi\|_{E_{0}}=\|\phi(c)-\xi(c)\|_{E}=\|(\phi-\xi)(c)\|_{E} .
$$

Observation II The Razumikhin class $\mathcal{R}_{c}$ of functions in $E_{0}$ is is not assumed to be topologically closed, so the sequence of successive iterations constructed as in the statement (a) converges to a PPF fixed point of the operator $T$ which may be outside of $\mathcal{R}_{c}$.

In this paper, we extend and generalize Theorems 2.2 and 2.4 to a pair of operators in Banach spaces and establish some interesting common fixed point theorems with PPF dependence. In the following section we prove our main PPF dependent common fixed point theorems for the operators satisfying different contractive conditions. 


\section{PPF Dependent Common Fixed Point Theory}

Let $S, T: E_{0} \rightarrow E$ be two operators. A point $\phi^{*} \in E_{0}$ is called a PPF dependent common fixed point of $S$ and $T$ if $S \phi^{*}=\phi^{*}(c)=T \phi^{*}$ for some $c \in I$ and any statement that guarantees existence of the PPF dependent common fixed points of the operators $S$ and $T$ is called a PPF dependent common fixed point theorem for the operators in Banach spaces.

We need the following definitions in what follows.

Definition 3.1. Two operators $S, T: E_{0} \rightarrow E$ is said to satisfy a condition of strong Cirić type generalized contraction if there exists a real number $0<\lambda<1$ satisfying

$$
\begin{aligned}
\|S \phi-T \xi\|_{E} \leq \lambda \max \left\{\|\phi(c)-\xi(c)\|_{E},\|\phi(c)-S \phi\|_{E},\|\xi(c)-T \xi\|_{E},\right. \\
\left.\frac{1}{2}\left[\|\phi(c)-T \xi\|_{E}+\|\xi(c)-S \phi\|_{E}\right]\right\}
\end{aligned}
$$

for all $\phi, \xi \in E_{0}$ and for some $c \in[a, b]$.

Definition 3.2. Two operators $S, T: E_{0} \rightarrow E$ is said to satisfy a condition of Cirić type generalized contraction if there exists a real number $0<\lambda<1$ satisfying

$$
\begin{aligned}
\|S \phi-T \xi\|_{E} \leq \lambda \max \left\{\|\phi-\xi\|_{E_{0}},\|\phi(c)-S \phi\|_{E},\|\xi(c)-T \xi\|_{E},\right. & \\
& \left.\frac{1}{2}\left[\|\phi(c)-T \xi\|_{E}+\|\xi(c)-S \phi\|_{E}\right]\right\}
\end{aligned}
$$

for all $\phi, \xi \in E_{0}$ and for some $c \in[a, b]$.

It is easy to see that every strong Cirić type generalized contraction is Cirić type generalized contraction, however the converse is necessarily not true.

Theorem 3.3. Suppose that $S, T: E_{0} \rightarrow E$ satisfy the condition of Ciric type generalized contraction. Then the following statements hold.

(a) If $\mathcal{R}_{c}$ is algebraically closed w.r.t the difference, then for a given $\phi_{0} \in E_{0}$ and $c \in[a, b]$, every sequence $\left\{\phi_{n}\right\}$ of iterates of $T$ defined by

$$
\begin{gathered}
S \phi_{2 n}=\phi_{2 n+1}(c), \quad T \phi_{2 n+1}=\phi_{2 n+2}(c) \\
\left\|\phi_{n}-\phi_{n+1}\right\|_{E_{0}}=\left\|\phi_{n}(c)-\phi_{n+1}(c)\right\|_{E}
\end{gathered}
$$

for $n=0,1,2, \ldots$, converges to a PPF dependent common fixed point of $S$ and $T$.

(b) If $\phi_{0}, \xi_{0} \in E_{0}$ and $\left\{\phi_{n}\right\},\left\{\xi_{n}\right\}$ are sequences defined by (3.3). Then,

$$
\left\|\phi_{n}-\xi_{n}\right\|_{E_{0}} \leq\left(\frac{1}{1-\lambda}\right)\left[\left\|\phi_{0}-\phi_{1}\right\|_{E_{0}}+\left\|\xi_{0}-\xi_{1}\right\|_{E_{0}}\right]+\left\|\phi_{0}-\xi_{0}\right\|_{E_{0}} .
$$

If, in particular $\phi_{0}=\xi_{0}$ and $\left\{\phi_{n}\right\} \not \equiv\left\{\xi_{n}\right\}$, then

$$
\left\|\phi_{n}-\xi_{n}\right\|_{E_{0}} \leq\left[\frac{2}{1-\lambda}\right]\left\|\phi_{0}-\xi_{0}\right\|_{E_{0}} .
$$

(c) If $\mathcal{R}_{c}$ is topologically closed, then $S$ and $T$ have a unique PPF dependent fixed point in $\mathcal{R}_{c}$. 
Proof. Let $\phi_{0} \in E_{0}$ be arbitrary and define a sequence $\left\{\phi_{n}\right\}$ in $E_{0}$ as follows. By hypothesis, $S \phi_{0} \in E$. Suppose that $S \phi_{0}=x_{1}$. Choose $\phi_{1} \in E_{0}$ such that $x_{1}=\phi_{1}(c)$ and $\left\|\phi_{1}-\phi_{0}\right\|_{E_{0}}=\left\|\phi_{1}(c)-\phi_{0}(c)\right\|_{E}$. Again, by hypothesis, $T \phi_{1} \in E$. Suppose that $T \phi_{1}=x_{2}$. Choose $\phi_{2} \in E_{0}$ such that $x_{2}=\phi_{2}(c)$ and $\left\|\phi_{2}-\phi_{1}\right\|_{E_{0}}=\left\|\phi_{2}(c)-\phi_{1}(c)\right\|_{E}$. Proceeding in this way, by induction, we obtain

$$
S \phi_{2 n}=\phi_{2 n+1}(c) ; T \phi_{2 n+1}=\phi_{2 n+2}(c)
$$

and

$$
\left\|\phi_{n}-\phi_{n+1}\right\|_{E_{0}}=\left\|\phi_{n}(c)-\phi_{n+1}(c)\right\|_{E}
$$

for all $n=0,1, \ldots$.

We claim that $\left\{\phi_{n}\right\}$ is a Cauchy sequence in $E_{0}$. Now for $n=0$, we have the following estimate:

$$
\begin{aligned}
\left\|\phi_{1}-\phi_{2}\right\|_{E_{0}}= & \left\|\phi_{1}(c)-\phi_{2}(c)\right\|_{E} \\
= & \left\|S \phi_{0}-T \phi_{1}\right\|_{E} \\
\leq & \lambda \max \left\{\left\|\phi_{0}-\phi_{1}\right\|_{E_{0}},\left\|\phi_{0}(c)-S \phi_{0}\right\|_{E},\left\|\phi_{1}(c)-T \phi_{1}\right\|_{E},\right. \\
& \left.\quad \frac{1}{2}\left[\left\|\phi_{0}(c)-T \phi_{1}\right\|_{E}+\left\|\phi_{1}(c)-S \phi_{0}\right\|_{E}\right]\right\} \\
\leq & \lambda \max \left\{\left\|\phi_{0}-\phi_{1}\right\|_{E_{0}},\left\|\phi_{0}(c)-\phi_{1}(c)\right\|_{E},\left\|\phi_{1}(c)-\phi_{2}(c)\right\|_{E},\right. \\
& \left.\frac{1}{2}\left[\left\|\phi_{0}(c)-\phi_{2}(c)\right\|_{E}+\left\|\phi_{1}(c)-\phi_{1}(c)\right\|_{E}\right]\right\} \\
& \leq \lambda \max \left\{\left\|\phi_{0}-\phi_{1}\right\|_{E_{0}},\left\|\phi_{0}-\phi_{1}\right\|_{E_{0}},\left\|\phi_{1}-\phi_{2}\right\|_{E_{0}},\right. \\
& \left.\frac{1}{2}\left[\left\|\phi_{0}-\phi_{2}\right\|_{E_{0}}+\left\|\phi_{1}-\phi_{1}\right\|_{E_{0}}\right]\right\} \\
\leq & \lambda \max \left\{\left\|\phi_{0}-\phi_{1}\right\|_{E_{0}},\left\|\phi_{1}-\phi_{2}\right\|_{E_{0}}, \frac{1}{2}\left[\left\|\phi_{0}-\phi_{2}\right\|_{E_{0}}\right\}\right. \\
\leq & \lambda \max \left\{\left\|\phi_{0}-\phi_{1}\right\|_{E_{0}}, \frac{1}{2}\left[\left\|\phi_{0}-\phi_{1}\right\|_{E_{0}}+\left\|\phi_{1}-\phi_{2}\right\|_{E_{0}}\right]\right\} \\
\leq & \lambda\left\|\phi_{0}-\phi_{1}\right\|_{E_{0}} .
\end{aligned}
$$

Similarly,

$$
\begin{aligned}
&\left\|\phi_{2}-\phi_{3}\right\|_{E_{0}}=\left\|\phi_{2}(c)-\phi_{3}(c)\right\|_{E} \\
&=\left\|S \phi_{2}-T \phi_{1}\right\|_{E} \\
& \leq \lambda \max \left\{\left\|\phi_{2}-\phi_{1}\right\|_{E_{0}},\left\|\phi_{2}(c)-S \phi_{2}\right\|_{E},\left\|\phi_{1}(c)-T \phi_{1}\right\|_{E},\right. \\
&\left.\quad \frac{1}{2}\left[\left\|\phi_{2}(c)-T \phi_{1}\right\|_{E}+\left\|\phi_{1}(c)-S \phi_{2}\right\|_{E}\right]\right\} \\
& \leq \lambda \max \left\{\left\|\phi_{2}-\phi_{1}\right\|_{E_{0}},\left\|\phi_{2}(c)-\phi_{3}(c)\right\|_{E},\left\|\phi_{1}(c)-\phi_{2}(c)\right\|_{E},\right. \\
&\left.\frac{1}{2}\left[\left\|\phi_{2}(c)-\phi_{2}(c)\right\|_{E}+\left\|\phi_{1}(c)-\phi_{3}(c)\right\|_{E}\right]\right\} \\
& \leq \lambda \max \left\{\left\|\phi_{2}-\phi_{1}\right\|_{E_{0}},\left\|\phi_{2}-\phi_{3}\right\|_{E_{0}},\left\|\phi_{1}-\phi_{2}\right\|_{E_{0}},\right. \\
&\left.\frac{1}{2}\left[\left\|\phi_{2}-\phi_{2}\right\|_{E_{0}}+\left\|\phi_{1}-\phi_{3}\right\|_{E_{0}}\right]\right\} \\
& \leq \lambda \max \left\{\left\|\phi_{1}-\phi_{2}\right\|_{E_{0}},\left\|\phi_{2}-\phi_{3}\right\|_{E_{0}}, \frac{1}{2}\left[\left\|\phi_{1}-\phi_{3}\right\|_{E_{0}}\right\}\right. \\
& \leq \lambda \max \left\{\left\|\phi_{1}-\phi_{2}\right\|_{E_{0}}, \frac{1}{2}\left[\left\|\phi_{1}-\phi_{2}\right\|_{E_{0}}+\left\|\phi_{2}-\phi_{3}\right\|_{E_{0}}\right]\right\} \\
& \leq \lambda\left\|\phi_{1}-\phi_{2}\right\|_{E_{0}} .
\end{aligned}
$$


Proceeding in this way, by induction y, we obtain

$$
\left\|\phi_{n}-\phi_{n+1}\right\|_{E_{0}} \leq \lambda\left\|\phi_{n-1}-\phi_{n}\right\|_{E_{0}}
$$

for all $n=1,2, \ldots$.

Hence, by repeated application of the above inequality yields

$$
\left\|\phi_{n}-\phi_{n+1}\right\|_{E_{0}} \leq \lambda^{n}\left\|\phi_{0}-\phi_{1}\right\|_{E_{0}}
$$

for all $n=1,2, \ldots$.

If $m>n$, by triangle inequality, we obtain

$$
\begin{aligned}
\left\|\phi_{m}-\phi_{n}\right\|_{E_{0}} & \leq\left\|\phi_{n}-\phi_{n+1}\right\|_{E_{0}}+\cdots+\left\|\phi_{m-1}-\phi_{m}\right\|_{E_{0}} \\
& \leq \lambda^{n}\left\|\phi_{0}-\phi_{1}\right\|_{E_{0}}+\cdots+\lambda^{m-1}\left\|\phi_{0}-\phi_{1}\right\|_{E_{0}} \\
& \leq\left(\lambda^{n}+\cdots+\lambda^{m-1}\right)\left\|\phi_{0}-\phi_{1}\right\|_{E_{0}} \\
& \leq \frac{\lambda^{n}}{1-\lambda}\left\|\phi_{0}-\phi_{1}\right\|_{E_{0}} .
\end{aligned}
$$

Hence,

$$
\lim _{m>n \rightarrow \infty}\left\|\phi_{m}-\phi_{n}\right\|_{E_{0}}=0
$$

As a result, the sequence $\left\{\phi_{n}\right\}$ is Cauchy. Since $E_{0}$ is complete, $\left\{\phi_{n}\right\}$ and every subsequence of it converges to a limit point $\phi^{*}$ in $E_{0}$, that is, $\lim _{n \rightarrow \infty} \phi_{n}=\phi^{*}$ and that $\lim _{n \rightarrow \infty} \phi_{2 n+1}=\phi^{*}=\lim _{n \rightarrow \infty} \phi_{2 n+2}$. We rove that $\phi^{*}$ is a PPF dependence fixed point of $T$.

Now, we first prove that $\phi^{*}$ is a PPF dependence fixed point of $S$. By inequality (3.1),

$$
\begin{aligned}
\| S \phi^{*}- & \phi^{*}(c)\left\|_{E} \leq\right\| S \phi^{*}-\phi_{2 n+2}(c)\left\|_{E}+\right\| \phi_{2 n+2}(c)-\phi^{*}(c) \|_{E} \\
\leq & \left\|S \phi^{*}-T \phi_{2 n+1}\right\|_{E}+\left\|\phi_{2 n+2}-\phi^{*}\right\|_{E_{0}} \\
\leq & \lambda \max \left\{\left\|\phi_{*}-\phi_{2 n+1}\right\|_{E_{0}},\left\|\phi^{*}(c)-S \phi^{*}\right\|_{E},\left\|\phi_{2 n+1}(c)-T \phi_{2 n+1}\right\|_{E}\right. \\
& \left.\frac{1}{2}\left[\left\|\phi^{*}(c)-T \phi_{2 n+1}\right\|_{E}+\left\|\phi_{2 n+1}(c)-S \phi^{*}\right\|_{E}\right]\right\}+\left\|\phi_{2 n+2}-\phi^{*}\right\|_{E_{0}} \\
\leq & \lambda \max \left\{\left\|\phi_{*}-\phi_{2 n+1}\right\|_{E_{0}},\left\|\phi^{*}(c)-S \phi^{*}\right\|_{E},\left\|\phi_{2 n+1}(c)-\phi_{2 n+2}(c)\right\|_{E}\right. \\
& \left.\frac{1}{2}\left[\left\|\phi^{*}(c)-\phi_{2 n+2}(c)\right\|_{E}+\left\|\phi_{2 n+1}(c)-S \phi^{*}\right\|_{E}\right]\right\}+\left\|\phi_{2 n+2}-\phi^{*}\right\|_{E_{0}} .
\end{aligned}
$$

Taking the limit superior as $n \rightarrow \infty$ in the above inequality yields,

$$
\left\|S \phi^{*}-\phi^{*}(c)\right\|_{E} \leq \lambda\left\|S \phi^{*}-\phi^{*}(c)\right\|_{E} .
$$

Hence, it follows that $S \phi^{*}=\phi^{*}(c)$. Similarly, it is proved that $T \phi^{*}=\phi^{*}(c)$. Then,

(b) Let $\phi_{0}, \xi_{0} \in E_{0}$ and let $\left\{\phi_{n}\right\}$ and $\left\{\xi_{n}\right\}$ be two sequences of iterations of $S$ and $T$ defined by (3.3).

$$
\begin{aligned}
\left\|\phi_{n}-\xi_{n}\right\|_{E_{0}} & \leq\left\|\phi_{n}-\phi_{n-1}\right\|_{E_{0}}+\left\|\phi_{n-1}-\xi_{n-1}\right\|_{E_{0}}+\left\|\phi_{n-1}-\xi_{n}\right\|_{E_{0}} \\
& \leq \lambda^{n}\left\|\phi_{0}-\phi_{1}\right\|_{E_{0}}+\left\|\phi_{n-1}-\xi_{n-1}\right\|_{E_{0}}+\lambda^{n}\left\|\xi_{0}-\xi_{1}\right\|_{E_{0}} \\
& \leq \lambda^{n}\left[\left\|\phi_{0}-\phi_{1}\right\|_{E_{0}}+\left\|\xi_{0}-\xi_{1}\right\|_{E_{0}}\right]+\left\|\phi_{n-1}-\xi_{n-1}\right\|_{E_{0}} \\
& \leq\left(\lambda^{n}+\cdots+1\right)\left[\left\|\phi_{0}-\phi_{1}\right\|_{E_{0}}+\left\|\xi_{0}-\xi_{1}\right\|_{E_{0}}\right]+\left\|\phi_{0}-\xi_{0}\right\|_{E_{0}} \\
& \leq \frac{1}{1-\lambda}\left[\left\|\phi_{0}-\phi_{1}\right\|_{E_{0}}+\left\|\xi_{0}-\xi_{1}\right\|_{E_{0}}\right]+\left\|\phi_{0}-\xi_{0}\right\|_{E_{0}} .
\end{aligned}
$$


In particular, if $\phi_{0}=\xi_{0}$, then $\phi_{0}(c)=\xi_{0}(c)$ so that $S \phi_{0}=S \xi_{0}$ and $\phi_{1}=\xi_{1}$. Hence, from inequality (3.4) it follows that

$$
\left\|\phi_{n}-\xi_{n}\right\|_{E_{0}} \leq \frac{2}{1-\lambda}\left\|\phi_{0}-\phi_{1}\right\|_{E_{0}}
$$

(c) To prove uniqueness of fixed point in $\mathcal{R}_{c}$, let $\phi^{*}$ and $\xi^{*}$ be two fixed points of $T$, then

$$
\begin{aligned}
\left\|\phi^{*}-\xi^{*}\right\|_{E_{0}}= & \left\|\phi^{*}(c)-\xi^{*}(c)\right\|_{E} \\
\leq & \left\|S \phi^{*}-T \xi^{*}\right\|_{E} \\
\leq & \lambda \max \left\{\left\|\phi^{*}-\xi^{*}\right\|_{E_{0}},\left\|\phi^{*}(c)-S \phi^{*}\right\|_{E},\left\|\xi^{*}(c)-T \xi^{*}\right\|_{E},\right. \\
& \left.\frac{1}{2}\left[\left\|\phi^{*}(c)-T \xi^{*}\right\|_{E}+\left\|\xi^{*}(c)-S \phi^{*}\right\|_{E}\right]\right\} \\
\leq & \lambda \max \left\{\left\|\phi^{*}-\xi^{*}\right\|_{E_{0}}, 0,0, \frac{1}{2}\left[\left\|\phi^{*}(c)-\xi^{*}(c)\right\|_{E}+\left\|\xi^{*}(c)-\phi^{*}(c)\right\|_{E}\right]\right\} \\
\leq & \lambda \max \left\{\left\|\phi^{*}-\xi^{*}\right\|_{E_{0}}, 0,0,\left\|\phi^{*}-\xi^{*}\right\|_{E_{0}}\right\}
\end{aligned}
$$

which yields $\phi^{*}=\xi^{*}$ since $\lambda<1$. This completes the proof.

On taking $S=T$ in (3.1), we obtain

Definition 3.4. An operator $T: E_{0} \rightarrow E$ is called a Cirić type generalized contraction if there exists a real number $0<\lambda<1$ satisfying

$$
\begin{aligned}
\|T \phi-T \xi\|_{E} \leq \lambda \max \left\{\|\phi-\xi\|_{E_{0}},\|\phi(c)-T \phi\|_{E},\|\xi(c)-T \xi\|_{E},\right. & \\
& \left.\frac{1}{2}\left[\|\phi(c)-T \xi\|_{E}+\|\xi(c)-T \phi\|_{E}\right]\right\}
\end{aligned}
$$

for all $\phi, \xi \in E_{0}$ and for some $c \in[a, b]$.

Remark 3.5. It is clear that contractions and strong Kannan type contractions are Ciric type generalized contractions, but the converse may not be true. The class of generalized contraction operators is supposed to be the most general one and includes several classes of contraction operators in metric spaces including those of Banach and Kanann etc. A nice comparison of different classes of contractive mappings appears in Rhoades [8].

As a special case of Theorem 3.3 we obtain the following corollary.

Corollary 3.6. Suppose that $T: E_{0} \rightarrow E$ is a generalized contraction. Then the following statements hold in $E_{0}$.

(a) If $\mathcal{R}_{c}$ is closed with respect to difference, then for a given $\phi_{0} \in E_{0}$, every sequence $\left\{\phi_{n}\right\}$ of iterates defined by 2.4 converges to a PPF dependent fixed point of $T$.

(b) If $\mathcal{R}_{c}$ is algebraically and topologically closed, then for a given $\phi_{0} \in E_{0}$ every sequence $\left\{\phi_{n}\right\}$ of iterates defined by (2.4) converges to a unique PPF dependent fixed point of $T$ in $\mathcal{R}_{c}$.

Proof. The proof is similar to Theorem 2.2 and hence we omit the details.

Remark 3.7. We note that operators in Theorems 3.3 and 3.6 are not required to satisfy any continuity condition on the domains of their definition.

Remark 3.8. Note that Corollary 3.6 includes Theorems 2.2 and 2.4 as special cases in view of Remark 3.7. 


\section{Existence of Coincidence Points with PPF Dependence}

We need the following definition in what follows.

Definition 4.1. Let $A: E_{0} \rightarrow E$ and $S: E_{0} \rightarrow E_{0}$ be two operators. A point $\phi^{*} \in E_{0}$ is called a PPF dependent coincidence point of $A$ and $S$ if $A \phi^{*}=S \phi^{*}(c)$ for some $c \in I$ and any mathematical statement that guarantees the existence of such a coincidence point is called a common coincident point theorem with PPF dependence.

We consider the following definitions in what follows.

Definition 4.2. Two operators $A: E_{0} \rightarrow E$ and $S: E_{0} \rightarrow E_{0}$ are said to satisfy a condition of strong Cirić type generalized contraction $(\mathrm{C})$ if there exists a real number $0<\lambda<1$ satisfying

$$
\begin{aligned}
\|A \phi-A \xi\|_{E} \leq \lambda \max \left\{\|S \phi(c)-S \xi(c)\|_{E},\|S \phi(c)-A \phi\|_{E},\|S \xi(c)-A \xi\|_{E},\right. & \\
& \left.\frac{1}{2}\left[\|S \phi(c)-A \xi\|_{E}+\|S \xi(c)-A \phi\|_{E}\right]\right\}
\end{aligned}
$$

for all $\phi, \xi \in E_{0}$ and for some $c \in[a, b]$.

Definition 4.3. Two operators $A: E_{0} \rightarrow E$ and $S: E_{0} \rightarrow E_{0}$ are said to satisfy a condition of Cirić type generalized contraction (C) if there exists a real number $0<\lambda<1$ satisfying

$$
\begin{aligned}
\|A \phi-A \xi\|_{E} \leq \lambda \max \left\{\|S \phi-S \xi\|_{E_{0}},\|S \phi(c)-A \phi\|_{E},\|S \xi(c)-A \xi\|_{E},\right. & \\
& \left.\frac{1}{2}\left[\|S \phi(c)-A \xi\|_{E}+\|S \xi(c)-A \phi\|_{E}\right]\right\}
\end{aligned}
$$

for all $\phi, \xi \in E_{0}$ and for some $c \in[a, b]$.

Our main coincident point theorem with PPF dependence is the following.

Theorem 4.4. Let $A: E_{0} \rightarrow E$ and $S: E_{0} \rightarrow E_{0}$ be two operators satisfying a Cirić type generalized contraction $(C)$. Further suppose that

(a) $A\left(E_{0}\right) \subset S\left(E_{0}\right)(c)$,

(b) $S\left(E_{0}\right)$ is complete, and

(c) $S$ is continuous.

If $\mathcal{R}_{c}$ is topologically and algebraically closed w.r.t the difference, then $A$ and $S$ have have a PPF dependent coincidence point in $\mathcal{R}_{c}$.

Proof. Let $\phi_{0} \in E_{0}$ be arbitrary and define a sequence $\left\{\xi_{n}\right\}$ in $E_{0}$ as follows. By hypothesis, $A \phi_{0} \in E$. Suppose that $A \phi_{0}=x_{1}$. Since $A\left(E_{0}\right) \subset S\left(E_{0}\right)(c)$, choose $\phi_{1} \in E_{0}$ such that $x_{1}=S \phi_{1}(c)=\xi_{1}(c)$ and $\left\|\xi_{1}-\xi_{0}\right\|_{E_{0}}=\left\|\xi_{1}(c)-\xi_{0}(c)\right\|_{E}$. Again, by hypothesis, $A \phi_{1} \in E$. Suppose that $A \phi_{1}=x_{2}$. Since $A\left(E_{0}\right) \subset$ $S\left(E_{0}\right)(c)$, choose $\phi_{2} \in E_{0}$ such that $x_{2}=S \phi_{2}(c)=\xi_{2}(c)$ and $\left\|\xi_{2}-\xi_{1}\right\|_{E_{0}}=\left\|\xi_{2}(c)-\xi_{1}(c)\right\|_{E}$. Proceeding in this way, by induction, we obtain

$$
\left.\begin{array}{c}
A \phi_{n}=S \phi_{n+1}(c), S \phi_{n+1}=\xi_{n+1} ; \\
\left\|\xi_{n}-\xi_{n+1}\right\|_{E_{0}}=\left\|\xi_{n}(c)-\xi_{n+1}(c)\right\|_{E}
\end{array}\right\}
$$

for all $n=0,1, \ldots$.

We claim that $\left\{\xi_{n}\right\}$ is a Cauchy sequence in $E_{0}$. Now for $n=0$, we have the following estimate:

$$
\left\|\xi_{1}-\xi_{2}\right\|_{E_{0}}=\left\|\xi_{1}(c)-\xi_{2}(c)\right\|_{E}
$$




$$
\begin{aligned}
& =\left\|A \phi_{0}-A \phi_{1}\right\|_{E} \\
& \leq \lambda \max \left\{\left\|S \phi_{0}-S \phi_{1}\right\|_{E_{0}},\left\|S \phi_{0}(c)-A \phi_{0}\right\|_{E},\left\|S \phi_{1}(c)-A \phi_{1}\right\|_{E},\right. \\
& \left.\quad \frac{1}{2}\left[\left\|S \phi_{0}(c)-A \phi_{1}\right\|_{E}+\left\|S \phi_{1}(c)-A \phi_{0}\right\|_{E}\right]\right\} \\
& \leq \lambda \max \left\{\left\|\xi_{0}-\xi_{1}\right\|_{E_{0}},\left\|\xi_{0}(c)-\xi_{1}(c)\right\|_{E},\left\|\phi_{1}(c)-\phi_{2}(c)\right\|_{E},\right. \\
& \left.\qquad \frac{1}{2}\left[\left\|\xi_{0}(c)-\xi_{2}(c)\right\|_{E}+\left\|\xi_{1}(c)-\xi_{1}(c)\right\|_{E}\right]\right\} \\
& \leq \lambda \max \left\{\left\|\xi_{0}-\xi_{1}\right\|_{E_{0}},\left\|\xi_{0}-\xi_{1}\right\|_{E_{0}},\left\|\xi_{1}-\xi_{2}\right\|_{E_{0}},\right. \\
& \left.\qquad \frac{1}{2}\left[\left\|\xi_{0}-\xi_{2}\right\|_{E_{0}}+\left\|\xi_{1}-\xi_{1}\right\|_{E_{0}}\right]\right\} \\
& \leq \lambda \max \left\{\left\|\xi_{0}-\xi_{1}\right\|_{E_{0}},\left\|\xi_{1}-\xi_{2}\right\|_{E_{0}}, \frac{1}{2}\left[\left\|\xi_{0}-\xi_{2}\right\|_{E_{0}}\right\}\right. \\
& \leq \lambda \max \left\{\left\|\xi_{0}-\xi_{1}\right\|_{E_{0}}, \frac{1}{2}\left[\left\|\xi_{0}-\xi_{1}\right\|_{E_{0}}+\left\|\xi_{1}-\xi_{2}\right\|_{E_{0}}\right]\right\} \\
& \leq \lambda\left\|\xi_{0}-\xi_{1}\right\|_{E_{0}} .
\end{aligned}
$$

Similarly,

$$
\begin{aligned}
\left\|\xi_{2}-\xi_{3}\right\|_{E_{0}} & =\left\|S \phi_{2}(c)-S \phi_{3}(c)\right\|_{E} \\
& =\left\|A \phi_{2}-A \phi_{1}\right\|_{E} \\
& \leq \lambda \max \left\{\left\|S \phi_{2}-S \phi_{1}\right\|_{E_{0}},\left\|S \phi_{2}(c)-A \phi_{2}\right\|_{E},\left\|S \phi_{1}(c)-A \phi_{1}\right\|_{E},\right. \\
& \left.\frac{1}{2}\left[\left\|S \phi_{2}(c)-A \phi_{1}\right\|_{E}+\left\|s \phi_{1}(c)-A \phi_{2}\right\|_{E}\right]\right\} \\
& \leq \lambda \max \left\{\left\|\xi_{2}-\xi_{1}\right\|_{E_{0}},\left\|\xi_{2}(c)-\xi_{3}(c)\right\|_{E},\left\|\xi_{1}(c)-\xi_{2}(c)\right\|_{E},\right. \\
& \left.\frac{1}{2}\left[\left\|\xi_{2}(c)-\xi_{2}(c)\right\|_{E}+\left\|\xi_{1}(c)-\xi_{3}(c)\right\|_{E}\right]\right\} \\
& \leq \lambda \max \left\{\left\|\xi_{2}-\xi_{1}\right\|_{E_{0}},\left\|\xi_{2}-\xi_{3}\right\|_{E_{0}},\left\|\xi_{1}-\xi_{2}\right\|_{E_{0}},\right. \\
& \left.\frac{1}{2}\left[\left\|\xi_{2}-\xi_{2}\right\|_{E_{0}}+\left\|\xi_{1}-\xi_{3}\right\|_{E_{0}}\right]\right\} \\
& \leq \lambda \max \left\{\left\|\xi_{1}-\xi_{2}\right\|_{E_{0}},\left\|\xi_{2}-\xi_{3}\right\|_{E_{0}}, \frac{1}{2}\left[\left\|\xi_{1}-\xi_{3}\right\|_{E_{0}}\right\}\right. \\
& \leq \lambda \max \left\{\left\|\xi_{1}-\xi_{2}\right\|_{E_{0}}, \frac{1}{2}\left[\left\|\xi_{1}-\xi_{2}\right\|_{E_{0}}+\left\|\xi_{2}-\xi_{3}\right\|_{E_{0}}\right]\right\} \\
& \leq \lambda\left\|\xi_{1}-\xi_{2}\right\|_{E_{0}} .
\end{aligned}
$$

Proceeding in this way, by induction, we obtain

$$
\left\|\xi_{n}-\xi_{n+1}\right\|_{E_{0}} \leq \lambda\left\|\xi_{n-1}-\xi_{n}\right\|_{E_{0}}
$$

for all $n=1,2, \ldots$.

Hence, by repeated application of the above inequality yields

$$
\left\|\xi_{n}-\xi_{n+1}\right\|_{E_{0}} \leq \lambda^{n}\left\|\xi_{0}-\xi_{1}\right\|_{E_{0}}
$$

for all $n=1,2, \ldots$.

If $m>n$, by triangle inequality, we obtain

$$
\left\|\xi_{m}-\xi_{n}\right\|_{E_{0}} \leq\left\|\xi_{n}-\xi_{n+1}\right\|_{E_{0}}+\cdots+\left\|\xi_{m-1}-\xi_{m}\right\|_{E_{0}}
$$




$$
\begin{aligned}
& \leq \lambda^{n}\left\|\xi_{0}-\xi_{1}\right\|_{E_{0}}+\cdots+\lambda^{m-1}\left\|\xi_{0}-\xi_{1}\right\|_{E_{0}} \\
& \leq\left(\lambda^{n}+\cdots+\lambda^{m-1}\right)\left\|\xi_{0}-\xi_{1}\right\|_{E_{0}} \\
& \leq \frac{\lambda^{n}}{1-\lambda}\left\|\xi_{0}-\xi_{1}\right\|_{E_{0}} .
\end{aligned}
$$

Hence,

$$
\lim _{m>n \rightarrow \infty}\left\|\xi_{m}-\xi_{n}\right\|_{E_{0}}=0
$$

As a result, the sequence $\left\{\xi_{n}\right\}$ is Cauchy. Since $E_{0}$ is complete, $\left\{\xi_{n}\right\}$ and every subsequence of it converges to a limit point $\xi^{*}$ in $E_{0}$, that is,

$$
\lim _{n \rightarrow \infty} \xi_{n}=\lim _{n \rightarrow \infty} S \phi_{n}=\xi^{*} \text { and } \lim _{n \rightarrow \infty} \xi_{n}(c)=\lim _{n \rightarrow \infty} A \phi_{n}=\xi^{*}(c) .
$$

From continuity of $S$ it follows that

$$
\xi^{*}=\lim _{n \rightarrow \infty} \xi_{n}=\lim _{n \rightarrow \infty} S \phi_{n}=S \lim _{n \rightarrow \infty} \phi_{n}=S \phi^{*} .
$$

We prove that $\phi^{*}$ is a PPF dependent coincidence point of $A$ and $S$. Suppose not. Then, by 4.2),

$$
\begin{aligned}
\| A \phi^{*}- & S \phi^{*}(c)\left\|_{E} \leq\right\| A \phi^{*}-A \phi_{n}\left\|_{E}+\right\| A \phi_{n}-S \phi^{*}(c) \|_{E} \\
& \leq\left\|A \phi^{*}-A \phi_{n}\right\|_{E}+\left\|S \phi_{n(c)}-S \phi^{*}(c)\right\|_{E} \\
& \leq \lambda \max \left\{\left\|S \phi^{*}-S \phi_{n}\right\|_{E_{0}},\left\|S \phi^{*}(c)-A \phi^{*}\right\|_{E},\left\|S \phi_{n}(c)-A \phi_{n}\right\|_{E},\right. \\
& \left.\frac{1}{2}\left[\left\|S \phi^{*}(c)-A \phi_{n}\right\|_{E}+\left\|s \phi_{n}(c)-A \phi^{*}\right\|_{E}\right]\right\} \\
& \leq \lambda \max \left\{\left\|\xi_{*}-\xi_{n}\right\|_{E_{0}},\left\|S \phi^{*}(c)-A \phi^{*}\right\|_{E},\left\|S \phi_{n}(c)-A \phi^{*}\right\|_{E},\right. \\
& \left.\frac{1}{2}\left[\left\|S \phi^{*}(c)-S \phi_{n}(c)\right\|_{E}+\left\|s \phi_{n}(c)-A \phi^{*}\right\|_{E}\right]\right\} \\
\leq & \lambda \max \left\{0,\left\|S \phi^{*}(c)-A \phi^{*}\right\|_{E}, 0, \frac{1}{2}\left[0+\left\|S \phi^{*}(c)-A \phi^{*}\right\|_{E}\right]\right\} \\
= & \lambda\left\|A \phi^{*}-S \phi^{*}(c)\right\|_{E}
\end{aligned}
$$

which is a contradiction since $0<\lambda<1$. Hence $A \phi^{*}=S \phi^{*}(c)$. Thus $\phi^{*}$ is a PPF dependent coincidence pint of $A$ and $S$. This completes the proof.

\section{Approximating PPF Dependent Common Fixed Points}

Given two operators $S, T: E_{0} \rightarrow E$, let $\mathcal{C F}(S, T)$ denote the class of all PPF dependent common fixed points of $S$ and $T$ in $E_{0}$, that is,

$$
\mathcal{C F}(S, T)=\left\{\phi \in E_{0}: S \phi^{*}=\phi^{*}(c)=T \phi^{*}\right\} .
$$

We consider the following definition in what follows

Definition 5.1. Two operators $S, T: E_{0} \rightarrow E$ are said to satisfy a condition of generalized nonexpansive if

$$
\begin{aligned}
\|S \phi-T \xi\|_{E} \leq \max \left\{\|\phi-\xi\|_{E_{0}}, \frac{1}{2}\left[\|\phi(c)-S \phi\|_{E}+\|\xi(c)-T \xi\|_{E}\right]\right. & \\
& \left.\frac{1}{2}\left[\|\phi(c)-T \xi\|_{E}+\|\xi(c)-S \phi\|_{E}\right]\right\}
\end{aligned}
$$

for all $\phi, \xi \in E_{0}$. 
In the following theorem we give a necessary and sufficient condition for the existence of a sequence which approximate the PPF dependent coincidence points.

Theorem 5.2. Suppose that $S, T: E_{0} \rightarrow E$ are generalized nonexpansive and that $\mathcal{C F}(S, T) \neq \emptyset$. Suppose that $\mathcal{R}_{c}$ is topologically and algebraically closed w.r.t the difference, and $\left\{\phi_{n}\right\}$ is a sequence of iterates of $S$ and $T$ defined as in Theorem 3.3 satisfying for some $c \in I$,

$$
\left\|\phi_{n}-\phi\right\|_{E_{0}}=\left\|\phi_{n}(c)-\phi(c)\right\|_{E}
$$

for all $\phi \in \mathcal{C F}(S, T)$. Then $\left\{\phi_{n}\right\}$ converges to a PPF dependent common fixed point of $S$ and $T$ if and only if

$$
\lim _{n \rightarrow \infty} d_{E_{0}}\left(\phi_{n}, \mathcal{C F}(S, T)\right)=0 .
$$

Proof. First we note that if $\lim _{n \rightarrow \infty} d_{E_{0}}\left(\phi_{n}, \mathcal{C F}(S, T)\right)=0$, then

$$
\lim _{n \rightarrow \infty} d_{E_{0}}\left(\phi_{2 n+1}, \mathcal{C F}(S, T)\right)=0 \text { and } \lim _{n \rightarrow \infty} d_{E_{0}}\left(\phi_{2 n+2}, \mathcal{C F}(S, T)\right)=0 .
$$

Similarly, for any $\phi^{*} \in \mathcal{C F}(S, T)$,

$$
\left\|S \phi-\phi^{*}(c)\right\|_{E} \leq\left\|\phi-\phi^{*}(c)\right\|_{E} \quad \text { and } \quad\left\|T \phi-\phi^{*}(c)\right\|_{E} \leq\left\|\phi-\phi^{*}(c)\right\|_{E}
$$

for all $\phi \in E_{0}$. We prove the theorem in two parts.

Necessary Part: Suppose that $\phi_{n} \rightarrow \phi^{*}$ for some $\phi^{*} \in \mathcal{C F}(S, T)$. Then,

$$
\lim _{n \rightarrow \infty} d_{E_{0}}\left(\phi_{n}, \mathcal{C F}(S, T)\right)=\lim _{n \rightarrow \infty}\left[\inf _{\phi \in \mathcal{C} \mathcal{F}(S, T)}\left\|\phi_{n}-\phi\right\|_{E_{0}}\right] \leq \lim _{n \rightarrow \infty}\left\|\phi_{n}-\phi^{*}\right\|_{E_{0}}=0 .
$$

Sufficient Part: Assume that $\lim _{n \rightarrow \infty} d_{E_{0}}\left(\phi_{n}, \mathcal{C F}(S, T)\right)=0$. Then for $\epsilon>0$, there exists an $n_{0} \in \mathbb{N}$ such that

$$
d_{E_{0}}\left(\phi_{n}, \mathcal{C F}(S, T)\right)<\frac{\epsilon}{2}
$$

for all $n \geq n_{0}$. We claim that $\left\{\phi_{n}\right\}$ is a Cauchy sequence in $E_{0}$. Now, for any $m>n \geq n_{0}$ one has

$$
\left\|\phi_{m}-\phi_{n}\right\|_{E_{0}} \leq\left\|\phi_{m}-\phi\right\|_{E_{0}}+\left\|\phi-\phi_{n}\right\|_{E_{0}}
$$

for all $\phi \in \mathcal{C F}(S, T)$. Consider the following estimate:

$$
\begin{aligned}
\|\| \phi_{2 m+1}-\phi \|_{E_{0}} & =\left\|\phi_{2 m+1}(c)-\phi(c)\right\|_{E} \\
& =\left\|S \phi_{2 m}-\phi(c)\right\|_{E} \\
& \leq\left\|\phi_{2 m}(c)-\phi(c)\right\|_{E} \\
& =\left\|T \phi_{2 m-1}-\phi(c)\right\|_{E} \\
& \leq\left\|\phi_{2 m-1}-\phi\right\|_{E_{0}} \\
& \vdots \\
& \leq\left\|\phi_{n_{0}}-\phi\right\|_{E_{0}}
\end{aligned}
$$

Again,

$$
\|\| \phi_{2 m+2}-\phi\left\|_{E_{0}} \leq\right\| \phi_{n_{0}}-\phi \|_{E_{0}}
$$

Since $m$ is arbitrary, one has

$$
\|\| \phi_{m}-\phi\left\|_{E_{0}} \leq\right\| \phi_{n_{0}}-\phi \|_{E_{0}}
$$

Similarly,

$$
\|\| \phi_{n}-\phi\left\|_{E_{0}} \leq\right\| \phi_{n_{0}}-\phi \|_{E_{0}}
$$


for all $\phi \in \mathcal{C F}(S, T)$. Hence, from (5.7), (5.8) and (5.9) it follows

$$
\|\| \phi_{m}-\phi_{n}\left\|_{E_{0}} \leq 2\right\| \phi_{n_{0}}-\phi \|_{E_{0}} .
$$

for all $\phi \in \mathcal{C F}(S, T)$. Taking infimum over $\mathcal{C F}(S, T)$, we obtain

$$
\|\| \phi_{m}-\phi_{n}\left\|_{E_{0}} \leq 2 \inf _{\phi \in \mathcal{C} \mathcal{F}(S, T)}\right\| \phi_{n_{0}}-\phi \|_{E_{0}}=2 d_{E_{0}}\left(\phi_{n_{0}}, \mathcal{C F}(S, T)\right)<\epsilon .
$$

Hence, $\left\{\phi_{n}\right\}$ is a Cauchy sequence in $E_{0}$. Since $E_{0}$ is complete, $\left\{\phi_{n}\right\}$ and every subsequence of it converges to a unique limit point, say $\phi^{*} \in E_{0}$. Now it can be proved as in the proof of Theorem 3.3 that $S \phi^{*}=\phi^{*}(c)=T \phi^{*}$. Thus $\phi^{*} \in \mathcal{C F}(S, T)$ and the proof of the theorem is complete.

If $S=T$ in $(5.1)$, we obtain

Definition 5.3. An operator $T: E_{0} \rightarrow E$ is said to be generalized nonexpansive if

$$
\begin{aligned}
\|T \phi-T \xi\|_{E} \leq \max \left\{\|\phi-\xi\|_{E_{0}}, \frac{1}{2}\left[\|\phi(c)-T \phi\|_{E}+\|\xi(c)-T \xi\|_{E}\right],\right. & \left.\frac{1}{2}\left[\|\phi(c)-T \xi\|_{E}+\|\xi(c)-T \phi\|_{E}\right]\right\}
\end{aligned}
$$

for all $\phi, \xi \in E_{0}$.

As s special case of Theorem 5.1, we obtain the following corollary.

Corollary 5.4. Suppose that $T: E_{0} \rightarrow E$ is generalized nonexpansive and that $\mathcal{F}(T) \neq \emptyset$. Suppose that Razumikhin class $\mathcal{R}_{c}$ of functions in $E_{0}$ is topologically and algebraically closed w.r.t the difference, and $\left\{\phi_{n}\right\}$ is a sequence of iterates of $T$ defined as in (2.4) satisfying for some $c \in I$,

$$
\left\|\phi_{n}-\phi\right\|_{E_{0}}=\left\|\phi_{n}(c)-\phi(c)\right\|_{E}
$$

for all $\phi \in \mathcal{F}(T)$, where $\mathcal{F}(T)$ is a set of all PPF dependent fixed points of $T$ in $E_{0}$. Then $\left\{\phi_{n}\right\}$ converges to a PPF dependent fixed point of $T$ if and only if

$$
\lim _{n \rightarrow \infty} d_{E_{0}}\left(\phi_{n}, \mathcal{F}(T)\right)=0 .
$$

Remark 5.5. We remark that Corollary 5.4 includes an approximating fixed point result of Bernfeld et al. 11 for quasi-nonexpansive operators in Banach spaces as a special case. Note that every generalized nonexpansive mapping is quasi-nonexpansive, however the converse may not be true.

\section{Conclusion}

Finally, we conclude this paper with the remark that common fixed point theorems with PPF dependence proved here are very fundamental in the fixed point theory involving geometric hypothesis of distance between the images and objects in question. However, using the principle that has been formulated in Theorems 3.3, 4.4 and 5.2, several other common fixed point theorems with PPF dependence for the operators with different domain and range spaces can be proved. The existence results of this paper may be extended to three or four operators in Banach spaces with appropriate medications. In a forthcoming paper, we plan to prove some PPF dependent random fixed point theorems for the pairs of operators satisfying generalized contractive conditions in separable Banach spaces on the lines of Dhage [5] via constructive method.

\section{Acknowledgements}

The author is thankful to the referee for giving some useful suggestions for the improvement of this paper. 


\section{References}

[1] S. R. Bernfeld, V. Lakshmikatham and Y. M. Reddy, Fixed point theorems of operators with PPF dependence in Banach spaces, Applicable Anal. 6 (1977), 271-280. 1, 2, 2, 2, $2.2,5.5$

[2] Lj. B. Cirić, Generalized contraction and fixed point theorems, Publ. Inst Math 12 (1971), 19-26.

[3] Lj. B. Cirić, A generalization of Banach's contraction principle, PAMS 45 (1974), 267-273.

[4] B. C. Dhage, Fixed point theorems with PPF dependence and functional differential equations, Fixed point Theory 12 (2011), (to appear). 1, 2, 2, 2, 2, 2.4

[5] B. C. Dhage, Some basic random fixed point theorems with PPF dependence and functional random differential equations, Diff. Equ. Appl. 4 (2012), 181195. 6

[6] R. Kannan, Some results on fixed points II, Amer. Math. Monthly 76 (1969), 405-408. 2

[7] W. V. Petryshyn and T. E. Williamson, Strong and weak convergence of the sequences of successive approximations for quasi-nonexpansive mappings, J. Math. Anal. Appl. 43 (1973), 459-497.

[8] B E. Rhoades, A comparison of various definitions of contractive mappings, Trans. AMS 226 (1977), 257-290. 3.5 